\title{
Furin inhibitor D6R suppresses epithelial-mesenchymal transition in SW1990 and PaTu8988 cells via the Hippo-YAP signaling pathway
}

\author{
MENG ZHOU ${ }^{1 *}$, YOULI ZHANG $^{1 *}$, HONG WEI $^{1}$, JUNBO HE $^{1}$, DAWEI WANG ${ }^{1}$, \\ BAODING $\mathrm{CHEN}^{2}$, JIAN ZENG ${ }^{3}$, AIHUA GONG ${ }^{3}$ and MIN XU ${ }^{1}$ \\ Departments of ${ }^{1}$ Gastroenterology and ${ }^{2}$ Ultrasound Diagnosis, Affiliated Hospital of Jiangsu University; \\ ${ }^{3}$ Department of Cell Biology, School of Medicine, Jiangsu University, Zhenjiang, Jiangsu 212013, P.R. China
}

Received September 25, 2016; Accepted October 3, 2017

DOI: $10.3892 / \mathrm{ol} .2017 .7672$

\begin{abstract}
Hexa-D-arginine (D6R), an inhibitor of furin, has potential therapeutic applications in different types of human tumor. However, the function of D6R in targeting pancreatic cancer cells remains to be elucidated. In the present study, the proliferation, invasion and migration abilities of SW1990 and PaTu8988 cells were examined using a Cell Counting Kit-8, and Transwell and wound healing assays. Subsequently, the expression of proteins associated with epithelial-mesenchymal transition (EMT) and the Hippo-yes-associated protein (YAP) pathway were detected using western blot analysis. It was revealed that D6R significantly inhibited the proliferation, migration and invasion abilities of SW1990 and PaTu8988 cells. Additionally, D6R led to the upregulation of E-cadherin (an epithelial marker), and the downregulation of $\mathrm{N}$-cadherin and vimentin (mesenchymal markers) in SW1990 and PaTu8988 cells. Furthermore, the results of the present study revealed that D6R significantly affected the YAP phosphorylation level and the total YAP protein level, indicating that D6R was functionally involved in the Hippo-YAP signaling pathway. It has been suggested that D6R-suppressed EMT in SW1990 and PaTu8988 cells may occur via the Hippo-YAP pathway and that it may be a feasible drug to ameliorate the malignant phenotype of SW1990 and PaTu8988 cells.
\end{abstract}

Correspondence to: Professor Aihua Gong, Department of Cell Biology, School of Medicine, Jiangsu University, 301 Xuefu Road, Zhenjiang, Jiangsu 212013, P.R. China

E-mail: ahg5@163.com

Dr Min Xu, Department of Gastroenterology, Affiliated Hospital of Jiangsu University, Jiangsu University, 301 Xuefu Road, Zhenjiang, Jiangsu 212013, P.R. China

E-mail: peterxu1974@163.com

${ }^{*}$ Contributed equally

Key words: hexa-D-arginine, epithelial-mesenchymal transition, furin, pancreatic cancer cells, Hippo-yes-associated protein

\section{Introduction}

Furin is a member of the pro-protein convertase (PC) family that activates precursor proteins by cleaving a specific recognition sequence, and has served an important function in the activation of bacterial toxins and viral glycoproteins, in addition to the metastatic progression of certain types of tumor $(1,2)$. It was revealed that furin may be a target for the development of potent and selective antiproteolytic agents, owing to the notable function of furin in the proteolytic activation of numerous pathogenic precursor proteins, including the pro-toxins of bacteria and viruses, such as influenza A, Ebola virus and anthrax infection (3-5). Furthermore, furin may process molecules associated with tumor aggression and metastatic potential, including transforming growth factor- $\beta$ (TGF- $\beta$ ), membrane type 1 matrix metalloproteinase (MMP) and vascular endothelial growth factor (6-8). Furin is required for the activation of numerous pathogenic precursor proteins and therefore, furin inhibition is a logical approach to inhibiting the activation of those proteins.

In previous studies, a variety of putative inhibitors of furin have been identified, the most attractive among these being small molecule compounds, including decanoyl-RVKR-chloromethylketone (CMK) (9), $\alpha 1$-antitrypsin Portland ( $\alpha 1$-PDX) (10), CCG 8294 (11) and hexa-D-arginine (D6R) (12). D6R, a type of small synthetic inhibitor, is less toxic and more effective compared with other small molecule compounds in vitro, including $\alpha 1-\mathrm{PDX}$, furin propeptide and proteinase inhibitor-8 (12), with inhibitory constant values for furin, PACE4 and PC1 being 0.106, 0.580 and $13.200 \mu \mathrm{M}$, respectively. It has been reported that D6R may be a treatment for bacterial and viral infections $(4,13,14)$. For example, D6R appeared to block the cleavage of pseudomonas aeruginosa exotoxin A in vitro and in vivo (15), to reduce hepatitis $\mathrm{B}$ e-antigen secretion in patients with chronic hepatitis $B$ viral infection and to facilitate the decrease of immune tolerance (9). However, little is known regarding the function of D6R in the progression of a tumor.

Pancreatic cancer is a highly fatal disease with a high mortality rate and a 5-year survival rate of $~ 5 \%$ (16). Pancreatic cancer lacks noticeable symptoms, progresses rapidly, and is characterized by early dissemination and poor prognosis (17). 
The present study revealed that D6R is able to suppress the proliferation and epithelial-mesenchymal transition (EMT) of pancreatic cancer cells. The results of the present study indicated that D6R may function as an ideal compound for anti-pancreatic cancer treatment.

\section{Materials and methods}

Cell culture. Pancreatic cancer SW1990 and PaTu8988 cell lines were obtained from the Second Military Medical University (Shanghai, China). Cells were cultured in Dulbecco's modified Eagle's medium (DMEM; Hyclone; GE Healthcare Life Sciences, Logan, UT, USA) with $10 \%$ fetal bovine serum (FBS; Invitrogen; Thermo Fisher Scientific, Inc, Waltham, MA, USA) at $37^{\circ} \mathrm{C}$ in a humidified incubator with a $5 \% \mathrm{CO}_{2}$ supply. Cells were treated with or without D6R (Fumeisi Biotechnology Co., Ltd., Nanjing, China) $(1 \mu \mathrm{g} / \mathrm{ml})$ for 48-72 h.

Cell proliferation assay. A Cell Counting Kit-8 (CCK-8, Beijing Solarbio Science \& Technology Co., Ltd., Beijing, China) was used to analyze cell viability. Briefly, cells were seeded onto a 96-well plate at 2,000 cells/well. A CCK-8 assay was used to assess the cells viability at the first, second, third, fourth and fifth day. Briefly, $10 \mu$ l CCK-8 was added to each well respectively and cells were incubated in the dark at $37^{\circ} \mathrm{C}$ for $2 \mathrm{~h}$, and absorbance measured at $490 \mathrm{~nm}$ with a iMark $^{\mathrm{TM}}$ microplate absorbance reader (Bio-Rad Laboratories, Inc., Hercules, CA, USA).

Colony formation assay. A colony formation assay was then used to detect the anchorage-independent growth of SW1990 and PaTu8988 cells. Cells were incubated in 6-well culture plates at 1,000 cells/well. The cell colonies were formed following incubation for 1-2 weeks, and then they were fixed with $4 \%$ paraformaldehyde (http://www.aladdin-e. com, Aladdin Shanghai Biochemical Technology Co., Ltd., Shanghai, China) for $20 \mathrm{~min}$ and stained with crystal violet (Aladdin Shanghai Biochemical Technology Co., Ltd.) for $30 \mathrm{~min}$ at room temperature. The numbers of colonies were counted and a graph was constructed.

Scratch wound healing assay. A scratch wound healing assay was used to detect the migration ability of SW1990 and PaTu8988 cells. Cells were seeded in a 24 -well plate at $2 \times 10^{5}$ cells/well and incubated for $6 \mathrm{~h}$, then a $10-\mu 1$ pipette tip was used to disrupt the confluent monolayer and the cell layer was washed with PBS three times. The width of scratch was visualized using a light microscope (magnification, $\mathrm{x} 4$; Olympus Corporation, Tokyo, Japan). Cells were then cultured in DMEM with or without $1 \mu \mathrm{g} / \mathrm{ml}$ D6R for $24 \mathrm{~h}$. The wounded monolayer was visualized using a light microscope (magnification, x4; Olympus Corporation, Tokyo, Japan).

Cell invasion assay. Cell invasion assays were performed using Transwell chambers (Corning, NY, USA) according to the manufacturer's protocol. A total of $4 \mu \mathrm{l} \mathrm{BD} \mathrm{Matrigel} \mathrm{Basement}$ Membrane matrix (BD Biosciences, Franklin Lakes, NJ, USA) was placed in each chamber. Cells were seeded at a density of $1 \times 10^{5}$ cells/well in Matrigel chambers in DMEM, and 10\% FBS was added to the lower chambers. Following incubation for $24 \mathrm{~h}$, with or without $1 \mu \mathrm{g} / \mathrm{ml}$ D6R, cells that remained on top of the filter were wiped off, and cells that had invaded to the lower chamber were stained and counted. Cells were fixed with $4 \%$ paraformaldehyde for $20 \mathrm{~min}$ and stained with $1 \%$ crystal violet for $30 \mathrm{~min}$ at room temperature. The numbers of invaded cells were counted and a graph was constructed.

Western blotting. Cells were rinsed with PBS 3 times on ice prior to treatment with radioimmunoprecipitation assay lysis buffer (Shanghai BioSun Sci\&Tech Co., Ltd., Shanghai, China) at $100^{\circ} \mathrm{C}$ for $10 \mathrm{~min}$. The mixture was then centrifuged at $4^{\circ} \mathrm{C}$ at 9,000 x g/min (Heal Force Development Ltd, Hong Kong, SAR, China) for $10 \mathrm{~min}$. The supernatant was removed and the total cellular protein concentration was measured using the BCA method. Approximately $30 \mu \mathrm{g}$ of protein was loaded in each lane and separated using SDS-PAGE (10\% gel) and transferred onto polyvinylidene fluoride membranes. Membranes were blocked with $5 \%$ non-fat milk at room temperature for $1 \mathrm{~h}$, then incubated with the primary antibodies at $4^{\circ} \mathrm{C}$ for $8 \mathrm{~h}$ and then secondary horseradish peroxidase (HRP)-conjugated antibodies at room temperature for $1 \mathrm{~h}$. Bands were detected using an enhanced chemiluminescence system (Minichemill, SageCreation, Beijing, China). Primary antibodies included; rabbit anti-Furin (1:500; cat no. 18413-1-AP; Proteintech Group, Inc., Chicago, IL, USA) and mouse anti- $\beta$-Tubulin (1:10,000, cat no. 6181), rabbit anti-N-Cadherin (1:1,000; cat no. 13116), rabbit anti-E-Cadherin (1:1,000; cat no. 3195), rabbit anti-Vimentin (1:1,000; cat no. 5741), rabbit anti-DBF2 kinase activator protein MOB1 (Mob1; 1:1,000, cat no. 13730), rabbit anti-p-Mob1 (1:1,000, cat no. 8699), rabbit anti-yes-associated protein (YAP; 1:1,000; cat no. 8418), rabbit anti-p-YAP (1:1,000, cat no. 13619) (all purchased from Cell Signaling Technology, Inc., Danvers, MA, USA). The secondary HRP-conjugated antibodies were goat anti-rabbit $\operatorname{IgG}(\mathrm{H}+\mathrm{L})$ secondary antibodies (1:10,000; cat. no. 31460; Invitrogen; Thermo Fisher Scientific) and goat anti-mouse $\operatorname{IgG}(\mathrm{H}+\mathrm{L})$ (1:5,000; cat. no. 31430; Invitrogen; Thermo Fisher Scientific).

Statistical analysis. All data were presented as the mean \pm the standard deviation. All statistical analyses were carried out using SPSS Statistical software (version 19; IBM Corp., Armonk, NY, USA). An unpaired Student's t-test was used to compare the significance between the experimental group and the control. $\mathrm{P}<0.05$ was considered to indicate a statistically significant difference.

\section{Results}

D6R inhibits the proliferation of SW1990 and PaTu8988 cells. CCK- 8 assays were used to examine the relative proliferation rates in SW1990 and PaTu8988 cells. As presented in Fig. 1, D6R treatment resulted in decreased relative rates of proliferation in SW1990 and PaTu8988 cells, indicating that D6R inhibited the proliferation of SW1990 and PaTu8988 cells. Furthermore, a colony-forming assay revealed that the number of colonies were $468 \pm 21$ and $173 \pm 14$ in the D6R-free and D6R treated groups of SW1990 cells, respectively, and 603 \pm 21 and $234 \pm 19$ in D6R-free and D6R-treated groups of PaTu8988 cells, respectively (Fig. 2). D6R treated cells demonstrated a significantly decreased number of colonies $(\mathrm{P}<0.05)$ for SW1990 and PaTu8988 cell lines compared with the untreated groups, 

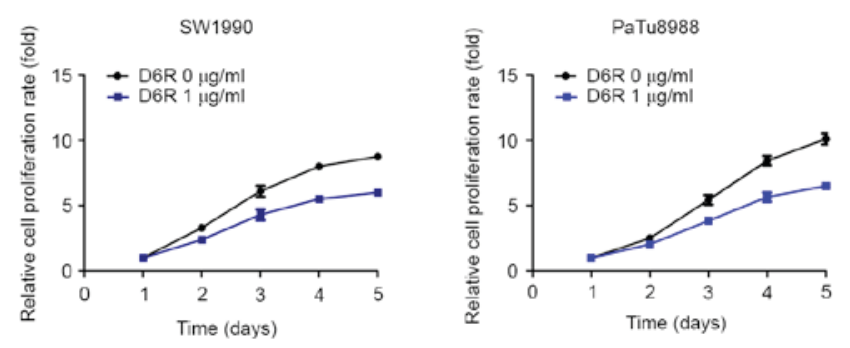

Figure 1. D6R treatment inhibits cell proliferation in SW1990 and PaTu8988 cells. A Cell Counting Kit-8 assay revealed that D6R inhibited the cell proliferation rate in SW1990 and PaTu8988 cells. D6R, hexa-D-arginine.
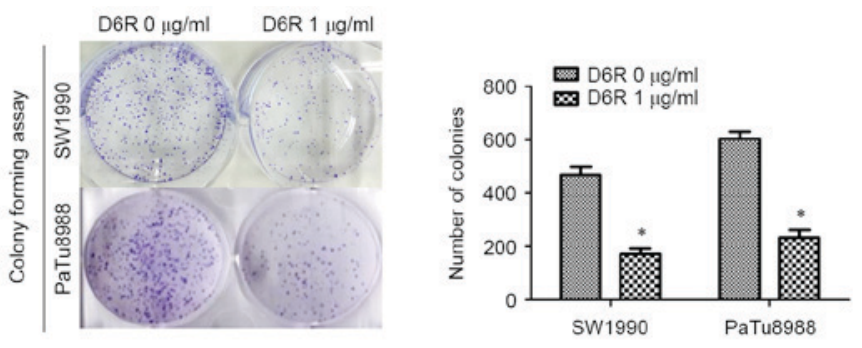

Figure 2. D6R inhibits anchorage-independent growth in SW1990 and PaTu8988 cells. A colony-forming assay revealed a significantly lower number of colonies formed in D6R treated cells compared with untreated cells for the two cell lines, revealing that D6R inhibited SW1990 and PaTu8988 cell anchorage-independent growth. ${ }^{*} \mathrm{P}<0.05$ vs. the $\mathrm{D} 6 \mathrm{R}$-free group. D6R, hexa-D-arginine.

suggesting that D6R suppresses anchorage-independent growth. This data suggests that D6R inhibited the proliferation ability of SW1990 and PaTu8988 cells.

D6R reduces cell invasiveness in SW1990 and PaTu 8988 cells. A Transwell invasion assay was used to examine the effect of D6R on the invasive abilities of SW1990 and PaTu8988 cells. As presented in Fig. 3, the numbers of invaded cells were $610 \pm 27$ and 306 \pm 15 in D6R-free and D6R treated groups of SW1990 cells, respectively, and 203 \pm 16 and $87 \pm 20$ in D6R-free and D6R treated groups of PaTu8988 cells, respectively. The number of invaded cells was significantly lower in the D6R treated groups for SW1990 and PaTu8988 cell lines $(\mathrm{P}<0.05)$ compared with the untreated groups. This data suggests that D6R inhibited the invasion ability of SW1990 and PaTu8988 cells.

D6R inhibits the migration ability of SW1990 and PaTu8988 cells. To determine the function of D6R in cell migration, a wound-healing assay was used to detect the migration ability of SW1990 and PaTu8988 cells. The width of the scratch at the beginning was $0.890 \pm 0.106$ and $1.043 \pm 0.210$ in D6R-free and D6R treated groups of SW1990 cells, respectively, and $0.893 \pm 0.182$ and $1.228 \pm 0.201$ in D6R-free and D6R treated groups of PaTu8988 cells. The migration rates were $0.864 \pm 0.011$ and $0.543 \pm 0.017$ in D6R-free and D6R treated groups of SW1990 cells, respectively, and $0.595 \pm 0.035$ and $0.450 \pm 0.020$ in D6R-free and D6R treated groups of PaTu8988 cells, respectively. The relative migration rate was significantly lower in the D6R treated groups of cells for SW1990 and PaTu8988 cell lines $(\mathrm{P}<0.05)$ compared with untreated groups.
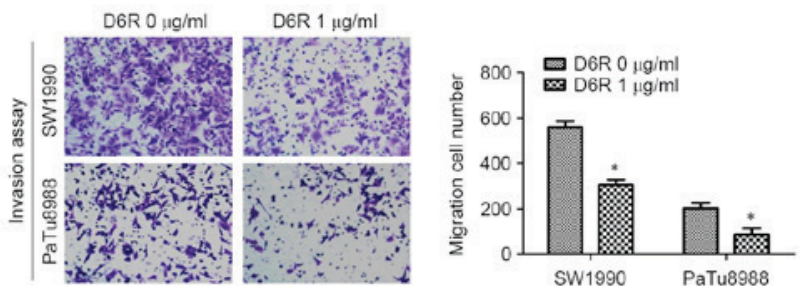

Figure 3. D6R reduced the cell invasiveness of SW1990 and PaTu8988 cells. Invasive ability was detected using a Transwell assay, and the migration cell number was significantly lower in the D6R treated cells compared with untreated cells for the two cell lines. ${ }^{*} \mathrm{P}<0.05$ vs. the D6R-free group. D6R, hexa-D-arginine. (magnification, x20).
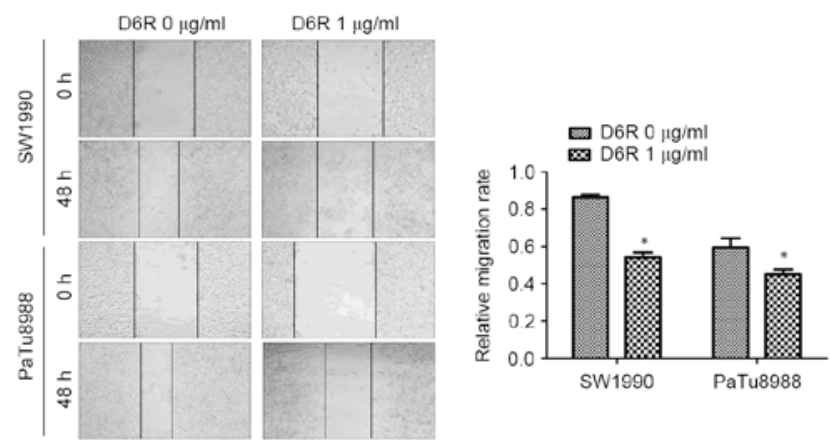

Figure 4. D6R inhibits the migration ability of SW1990 and PaTu8988 cells. Migration ability was detected using a wound healing assay, and the relative migration rate was significantly lower in the D6R treated cells compared with untreated cells for the two cell lines. ${ }^{*} \mathrm{P}<0.05$ vs. the D6R-free group. D6R, hexa-D-arginine.
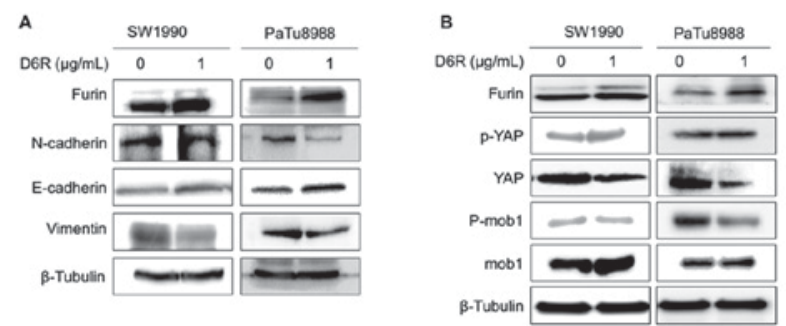

Figure 5. D6R affects the epithelial-mesenchymal transition and the Hippo-YAP pathway in SW1990 and PaTu8988 cells. (A) The protein levels of E-cadherin, N-cadherin and vimentin were measured using western blotting. (B) The protein levels of YAP, p-YAP, Mob1 and p-Mob1 were measured using western blotting. D6R, hexa-D-arginine; YAP, yes-associated protein; Mob1, DBF2 kinase activator protein MOB1; p, phosphorylated.

These results suggest that D6R inhibited the migration ability of SW1990 and PaTu8988 cells (Fig. 4).

D6R inhibits EMT in SW1990 and PaTu8988 cells. EMT serves a key function in allowing primary tumor cells to be capable of metastasizing. To determine whether D6R affects EMT, the expression of EMT relative proteins were examined with or without D6R treatment. Fig. 5A demonstrates that D6R resulted in the downregulation of $\mathrm{N}$-cadherin and vimentin, and the upregulation of E-cadherin in SW1990 and PaTu8988 cells. Mature furin expression was slightly increased in cells treated with D6R, thus suggesting that D6R suppressed the activity of furin, and may have caused to some extent an 
accumulation of the enzyme. This data confirms that D6R suppressed EMT in SW1990 and PaTu8988 cells.

D6R affects the Hippo-YAP pathway in SW1990 and PaTu8988 cells. It has been reported that Hippo-YAP signaling is associated with EMT $(2,6,7)$. To explore whether the Hippo-YAP pathway reacted to D6R inhibiting EMT in SW1990 and PaTu8988 cells, the expression of the relevant proteins in the Hippo-YAP pathway was examined with or without D6R treatment in SW1990 and PaTu8988 cells. The results of the present study revealed that D6R resulted in the downregulation of total YAP and p-Mob1, and the upregulation of p-YAP and Mob1 protein levels. This data suggests that D6R affected the Hippo-YAP signaling pathway in SW1990 and PaTu8988 cells (Fig. 5B).

\section{Discussion}

In the present study, it was identified that D6R, functioning as a furin inhibitor, suppressed the proliferation, migration and invasion of SW1990 and PaTu8988 cells, and characterized furin as an oncogene. Notably, D6R inhibited EMT potentially via the Hippo-YAP signaling pathway.

Differing types of small molecule components, including a1-PDX, Furin propeptide and proteinase inhibitor- 8 , which function as competitive furin inhibitors, have been well characterized. A number of them demonstrated the potential to be used for the treatment of certain infections, including bacterial and viral infections, such as Pseudomonas aeruginosa exotoxin A, Bacillus anthraci and Hepatitis B $(4,13,14)$. Additionally, a number of them have been reported to reduce the growth and invasiveness of numerous types of tumor cells $(10,11,18,19)$. $\alpha 1$-PDX resulted in the reduction of the growth and invasive ability, and malignant phenotypes of HT-29 human colon carcinoma cells (18), glioma tumor cells (20) and head and neck squamous cell carcinoma cells (10). A small-molecule inhibitor of furin named CCG 8294 and decRVKR-CMK inhibited the maturation of MMPs and the invasiveness of human fibrosarcoma cells $(11,21)$. However, collective studies have demonstrated the limitations of these inhibitors. It has been revealed that polyarginines were characterized by high potency, specificity and low toxicity $(12,22)$, compared with other small molecules. Previous studies have demonstrated the potency of D6R treatment on viral and bacterium infections, but little is known about the anti-metastatic potential of D6R. Therefore, the migration and invasion potential were measured using treatments of D6R at a concentration of $1 \mu \mathrm{g} / \mathrm{ml}$ in SW1990 and PaTu8988 cells. It was demonstrated that D6R inhibited the migration and invasion ability and EMT in SW1990 and PaTu8988 cells.

EMT, a crucial cellular mechanism in tumor metastasis, is a developmental process in which cells lose their epithelial features and develop a mesenchymal phenotype allowing cells to escape and spread to distant sites (23). Accumulating evidence indicates that substrate molecules processed by furin, including MMPs and TGF- $\beta$, are critical for the promotion of EMT, which contributes to cancer metastasis $(6,7,24-26)$. From the results of the present study, it may be concluded that D6R suppressed EMT and served a crucial function in pancreatic cancer metastasis. In the present study, it was confirmed that D6R treatment resulted in the downregulation of $\mathrm{N}$-cadherin and vimentin, and the upregulation of E-cadherin, consistent with the reduction in the invasion and migration ability of SW1990 and PaTu8988 cells. Furthermore, it was conclusively demonstrated that D6R inhibited EMT in line with alterations in YAP phosphorylation levels and the total YAP protein level, suggesting that YAP was involved in the regulation of EMT suppressed by D6R in SW1990 and PaTu8988 cells. Despite these notable findings, further investigations are required to elucidate the mechanism of D6R in the regulation of EMT via the Hippo-YAP pathway.

In summary, the present study indicates that D6R reduced the proliferation, migration and invasive ability of SW1990 and PaTu8988 cells, and that D6R-suppressed EMT may be regulated via the Hippo-YAP signaling pathway. Altogether, D6R has the potential to be used as a drug candidate to ameliorate a malignant phenotype in SW1990 and PaTu8988 cells.

\section{Acknowledgements}

The present study was supported by the National Natural Science Foundation of China (grant nos. 81672402, 81472333 and 81372718) and the Natural Science Foundation of Jiangsu Province (grant no. BK20131247).

\section{References}

1. Bassi DE, Mahloogi H and Klein-Szanto AJ: The proprotein convertases furin and PACE4 play a significant role in tumor progression. Mol Carcinog 28: 63-69, 2000.

2. Pei D and Weiss SJ: Furin-dependent intracellular activation of the human stromelysin-3 zymogen. Nature 375: 244-247, 1995.

3. Shiryaev SA, Remacle AG, Ratnikov BI, Nelson NA, Savinov AY Wei G, Bottini M, Rega MF, Parent A, Desjardins R, et al: Targeting host cell furin proprotein convertases as a therapeutic strategy against bacterial toxins and viral pathogens. J Biol Chem 282: 20847-20853, 2007.

4. Becker GL, Lu Y, Hardes K, Strehlow B, Levesque C, Lindberg I, Sandvig K, Bakowsky U, Day R, Garten W and Steinmetzer T: Highly potent inhibitors of proprotein convertase furin as potential drugs for treatment of infectious diseases. J Biol Chem 287: 21992-22003, 2012.

5. Thomas G: Furin at the cutting edge: From protein traffic to embryogenesis and disease. Nat Rev Mol Cell Biol 3: 753-766, 2002.

6. Dubois CM, Blanchette F, Laprise MH, Leduc R, Grondin F and Seidah NG: Evidence that furin is an authentic transforming growth factor-betal-converting enzyme. Am J Pathol 158: 305-316, 2001.

7. Yana I and Weiss SJ: Regulation of membrane type-1 matrix metalloproteinase activation by proprotein convertases. Mol Biol Cell 11: 2387-2401, 2000.

8. López de Cicco R, Watson JC, Bassi DE, Litwin S and Klein-Szanto AJ: Simultaneous expression of furin and vascular endothelial growth factor in human oral tongue squamous cell carcinoma progression. Clin Cancer Res 10: 4480-4488, 2004.

9. Pang YJ, Tan XJ, Li DM, Zheng ZH, Lei RX and Peng XM: Therapeutic potential of furin inhibitors for the chronic infection of hepatitis B virus. Liver Int 33: 1230-1238, 2013.

10. Bassi DE, Lopez De Cicco R, Mahloogi H, Zucker S, Thomas G and Klein-Szanto AJ: Furin inhibition results in absent or decreased invasiveness and tumorigenicity of human cancer cells. Proc Natl Acad Sci USA 98: 10326-10331, 2001.

11. Coppola JM, Bhojani MS, Ross BD and Rehemtulla A: A small-molecule furin inhibitor inhibits cancer cell motility and invasiveness. Neoplasia 10: 363-370, 2008.

12. Cameron A, Appel J, Houghten RA and Lindberg I: Polyarginines are potent furin inhibitors. J Biol Chem 275: 36741-36749, 2000. 
13. Komiyama T, Swanson JA and Fuller RS: Protection from anthrax toxin-mediated killing of macrophages by the combined effects of furin inhibitors and chloroquine. Antimicrob Agents Chemother 49: 3875-3882, 2005.

14. Ozden S, Lucas-Hourani M, Ceccaldi PE, Basak A, Valentine M Benjannet S, Hamelin J, Jacob Y, Mamchaoui K, Mouly V, et al: Inhibition of Chikungunya virus infection in cultured human muscle cells by furin inhibitors: Impairment of the maturation of the E2 surface glycoprotein. J Biol Chem 283: 21899-21908, 2008.

15. Sarac MS, Cameron A and Lindberg I: The furin inhibitor hexa-D-arginine blocks the activation of Pseudomonas aeruginosa exotoxin A in vivo. Infect Immun 70: 7136-7139, 2002.

16. Wolfgang CL, Herman JM, Laheru DA, Klein AP, Erdek MA, Fishman EK and Hruban RH: Recent progress in pancreatic cancer. CA Cancer J Clin 63: 318-348, 2013.

17. Reubi JC: Peptide receptors as molecular targets for cancer diagnosis and therapy. Endocr Rev 24: 389-427, 2003.

18. Khatib AM, Siegfried G, Prat A, Luis J, Chrétien M, Metrakos P and Seidah NG: Inhibition of proprotein convertases is associated with loss of growth and tumorigenicity of HT-29 human colon carcinoma cells: Importance of insulin-like growth factor-1 (IGF-1) receptor processing in IGF-1-mediated functions. J Biol Chem 276: 30686-30693, 2001.

19. Longuespée R, Couture F, Levesque C, Kwiatkowska A, Desjardins R, Gagnon S, Vergara D, Maffia M, Fournier I, Salzet M and Day R: Implications of Proprotein Convertases in Ovarian Cancer Cell Proliferation and Tumor Progression: Insights for PACE4 as a Therapeutic Target. Transl Oncol: pii:S1936-5233, 2014.
20. Mercapide J, Lopez De Cicco R, Bassi DE, Castresana JS, Thomas G and Klein-Szanto AJ: Inhibition of furin-mediated processing results in suppression of astrocytoma cell growth and invasiveness. Clin Cancer Res 8: 1740-1746, 2002.

21. Maquoi E, Noël A, Frankenne F, Angliker H, Murphy G and Foidart JM: Inhibition of matrix metalloproteinase 2 maturation and HT1080 invasiveness by a synthetic furin inhibitor. FEBS Lett 424: 262-266, 1998.

22. Angliker H: Synthesis of tight binding inhibitors and their action on the proprotein-processing enzyme furin. J Med Chem 38: 4014-4018, 1995

23. Bartholin L: Pancreatic cancer and the tumor microenvironment: Mesenchyme's role in pancreatic carcinogenesis. In: Pancreatic Cancer and Tumor Microenvironment. Grippo PJ and Munshi HG, (eds.). Trivandrum (India), 2012.

24. Rasheed ZA, Yang J, Wang Q, Kowalski J, Freed I, Murter C, Hong SM, Koorstra JB, Rajeshkumar NV, He X, et al: Prognostic significance of tumorigenic cells with mesenchymal features in pancreatic adenocarcinoma. J Natl Cancer Inst 102: 340-351, 2010.

25. Hotz B, Arndt M, Dullat S, Bhargava S, Buhr HJ and Hotz HG: Epithelial to mesenchymal transition: Expression of the regulators snail, slug, and twist in pancreatic cancer. Clin Cancer Res 13: 4769-4776, 2007.

26. Arumugam T, Ramachandran V, Fournier KF, Wang $H$, Marquis L, Abbruzzese JL, Gallick GE, Logsdon CD, McConkey DJ and Choi W: Epithelial to mesenchymal transition contributes to drug resistance in pancreatic cancer. Cancer Res 69: 5820-5828, 2009. 